\title{
Ritz variational method for bending of rectangular kirchhoff plate under transverse hydrostatic load distribution
}

\author{
Clifford U. Nwoji ${ }^{1}$, Hyginus N. Onah ${ }^{1}$, Benjamin O. Mama ${ }^{1}$, Charles C. Ike ${ }^{2 *}$ \\ ${ }^{1}$ Dept of Civil Engineering,University of Nigeria, Nsukka, Enugu State, Nigeria \\ ${ }^{2}$ Dept of Civil Engineering,Enugu State University of Science \& Technology, Enugu State, Nigeria
}

Corresponding Author Email: ikecc2007@yahoo.com

https://doi.org/10.18280/mmep.050101

Received: 12 Feburary 2018

Accepted: 15 March 2018

\section{Keywords:}

Ritz variational method, Kirchhoff

plate, hydrostatic load distribution

\begin{abstract}
In this study, the Ritz variational method was used to analyze and solve the bending problem of simply supported rectangular Kirchhoff plate subject to transverse hydrostatic load distribution over the entire plate domain. The deflection function was chosen based on double series of infinite terms as coordinate function that satisfy the geometric and force boundary conditions and unknown generalized displacement parameters. Upon substitution into the total potential energy functional for homogeneous, isotropic Kirchhoff plates, and evaluation of the integrals, the total potential energy functional was obtained in terms of the unknown generalized displacement parameters. The principle of minimization of the total potential energy was then applied to determine the unknown displacement parameters. Moment curvature relations were used to find the bending moments. It was found that the deflection functions and the bending moment functions obtained for the plate domain, and the values at the plate center were exactly identical as the solutions obtained by Timoshenko and Woinowsky-Krieger using the Navier series method.
\end{abstract}

\section{INTRODUCTION}

Plates are commonly applied in many engineering fields as roof slabs, building floor slabs, bridge deck slabs, foundation footings, water tanks, bulkheads, retaining walls, turbine disks, aerospacecraft panels, and ship hulls [1, 2, 3]. Their applications cut across the various branches of engineering and plates are used in civil, structural, marine, naval, aerospace and mechanical engineering.

Plates are subjected to transversely applied loads, causing flexure and the development of flexural deformation and stresses. Plates are defined by their shapes as rectangular, square, circular, elliptical, skew, quadrilateral plates, etc. They are also defined by: their constituent materials as homogeneous, non homogeneous, isotropic, anisotropic, and orthotropic [1, 2, 3]. They are also classified as membranes, thin plates, moderately thick plates, and thick plates, depending on the ratio $(h / a)$ of their thickness, $h$ to the least inplane dimension, $a$.

Plates can be subjected to static loads or dynamic loads, and can also be subjected to inplane compressive loads that cause buckling. Thus, plates can respond to applied loads by static flexural response, dynamic flexural response or by buckling; depending on whether the loads are static or dynamic or compressive.

\section{Research aim and objectives}

The research aim is to apply the Ritz variational method to the flexural analysis of simply supported Kirchhoff plate under transverse hydrostatic load distribution over the entire plate domain. The objectives include: (i) to determine the total potential energy functional for the simply supported Kirchhoff plate under transverse hydrostatic load distribution for a suitable choice of displacement basis function

(ii) to apply the variational principle to the total potential energy functional determined and hence find the unknown displacement parameters which minimize the total potential energy functional

(iii) to determine the bending moment distributions from the bending moment curvature relations, and find the bending moments at the center of the plate

(iv) to find the deflection at the center of the plate.

\section{LITERATURE REVIEW}

\section{Review of plate theories}

Sophie Germain used the method of variational calculus to derive from the first principles, the differential equation governing thin plates subject to transverse distributed loads. Her differential equation was however defective, as she neglected the contributions to the strain energy due to the twisting deformations of the plate middle surface. Lagrange later obtained the corrected governing differential equation for transversely loaded thin plates that accounted for the warping (twisting) of the plate middle surface. Subsequently, Navier, [4] using the theory of elasticity, obtained the differential equation of bending of rectangular plates under distributed transverse loads. Navier [4] also used the Fourier double trigonometric series method to solve and obtain exact 
solutions for the deflection of simply supported rectangular thin plates under transverse loads.

Poisson [5] [6] extended Navier's research to the problem of circular plates. Kirchhoff [7] presented an extended theory of plates which considered both the transverse distributed loads and the in-plane loads on plates. Kirchhoff also applied the method of virtual displacements in solving plate problems. Hencky [8, 9] and Reissner [10, 11] have presented improvements to the Kirchhoff plate theory which considered the effect of transverse shear deformation on the behaviour of plates, and thus allow their application to the problem of moderately thick and thick plates. Their theories are first order shear deformation plate theories.

Mindlin [12] used a displacement based formulation to obtain the governing differential equation for first order shear deformable plates, and incorporated the effect of rotary inertia. Mindlin's theory, however, requires a shear correction factor to account for the error introduced by the assumption of a constant shear strain through the plate thickness.

Other plate models and theories that have been developed to account for the limitations and imperfections of the classical Kirchhoff-Love plate theory include: Shimpi refined plate theory [13], Higher Order Plate Deformation theory [14], Reddy's third order plate theory [15], Leung's plate theory [16], Osadebe plate model [17], and modified plate theories [18].

The plate problem is generally, a boundary value problem which is a system of differential equations which are required to be satisfied in the plate domain and the associated boundary conditions to be satisfied at the plate boundaries [19]. The plate problem has been successfully solved in the technical literature using two fundamental methods; namely: analytical closed form methods and numerical or approximate methods.

Ladeveze [20] introduced a new approach for the analysis and solution of homogeneous, isotropic elastic plates with constant thickness under arbitrary distributed flexural loads. His theory was described as exact because it lead to exact values of the generalized two dimensional quantities. Contrary to classical plate theories, Ladeveze's approach was not limited to thin plates. Analytical closed form methods that have been used in solving the plate problem include the double trigonometric series method by Navier [4], the single trigonometric series method by Levy [21]; closed form solutions have also been obtained by Nadai [22].

Other researchers that have presented closed form mathematical solutions to the plate problem include Mama et al [23] who used the finite Fourier sine transform method to solve the problem of simply supported rectangular Kirchhoff plates under uniformly distributed transverse loads over the entire plate surface, uniformly distributed patch load over a given area of the plate, and point load on the plate. Numerical methods have also been used to find approximate solutions to the plate bending problem. Nwoji et al [24] used the Kantorovich-Vlasov method to solve the simply supported plate problem for the case of uniformly distributed transverse load applied over the entire plate surface. Osadebe et al [25] applied the Galerkin-Vlasov method to the flexural analysis of simply supported Kirchhoff plates under uniformly distributed transverse loads applied on the entire plate under region. Nwoji et al [26] applied the Galerkin-Vlasov method to the flexural analysis of rectangular Kirchhoff plates with clamped and simply supported edges for the case of uniformly distributed transverse loads. Ike [27] used the KantorovichEuler-Lagrange-Galerkin's method to solve the flexural problem of Kirchhoff plates with clamped and simply supported edges. Other researchers who have worked on the plate problem include Mama et al [28], Ezeh et al [29] and Aginam et al [30].

\section{RITZ VARIATIONAL METHOD}

The Ritz variational method for the flexural analysis of the Kirchhoff plate under given applied loads and edge support conditions is based on the application of the principle of stationary total potential energy. The principle of minimum total potential energy states that the displacement field corresponding to the minimum total potential energy functional of the structure under applied loads represents a state of equilibrium provided the displacement field satisfies the prescribed boundary conditions of the structure. Hence, for the plate flexure problem, the Ritz variational method seeks to determine the displacement field $w(x, y)$ defined over the plate domain, where $x$ and $y$ are the plate domain coordinates and $w(x, y)$ is the displacement function or the displacement field, such that the geometric and force boundary conditions are satisfied and the total potential energy functional, $\Pi$ of the Kirchhoff plate, which is the sum of the stain energy in bending and the potential of the external distributed load is minimized.

Ritz assumed the displacement field $w(x, y)$ in terms of a linear combination of products of basis (shape or coordinate) functions of the plate in the $x$ and $y$ coordinate directions chosen such that these shape (basis or coordinate) functions satisfy apriori the end conditions along the $x$ and $y$ coordinate directions. The Ritz deflection field $w(x, y)$ for plates is generally given by the double series of infinite terms:

$$
w(x, y)=\sum_{m=1}^{\infty} \sum_{n=1}^{\infty} w_{m n} X_{m}(x) Y_{n}(y)
$$

where $X_{m}(x)$ and $Y_{n}(y)$ are the basis (coordinate or shape) functions in the $x$ and $y$ coordinate directions, respectively; and $w_{m n}$ are unknown parameters of the displacement field, which are sought. $w_{m n}$ are called generalized displacement parameters.

For Kirchhoff plates under transversely applied static loads, the total potential energy functional for small deformation assumptions and linear elastic, isotropic and homogeneous, material properties, becomes in terms of the displacement field:

$$
\begin{aligned}
& \Pi=\frac{D}{2} \iint_{R}\left[\left(\sum_{m}^{\infty} \sum_{n}^{\infty} w_{m n} X_{m}^{\prime \prime} Y_{n}+\sum_{m}^{\infty} \sum_{n}^{\infty} w_{m n} X_{m} Y_{n}^{\prime \prime}\right)^{2}+2(1-\mu)\left(\sum_{m}^{\infty} \sum_{n}^{\infty} w_{m n} X_{m}^{\prime} Y_{n}^{\prime}-\right.\right. \\
& \left.\left(\sum_{m}^{\infty} \sum_{n}^{\infty} w_{m n} X_{m}^{\prime \prime} Y_{n}\right)\left(\sum_{m}^{\infty} \sum_{n}^{\infty} w_{m n} X_{m} Y_{n}^{\prime \prime}\right)\right] d x d y \\
& -\iint_{R} p(x, y) \sum_{m}^{\infty} \sum_{n}^{\infty} w_{m n} X_{m} Y_{n} d x d y
\end{aligned}
$$

where the primes denote space derivatives of the basis functions.

Applying the principle of stationary total potential energy, Ritz variational method requires that for equilibrium, the total potential energy functional $\Pi$ should be minimized with respect to the unknown displacement field. Hence, for an 
extremum of $\Pi$ with respect to $w_{m n}$, the following condition is imposed:

$$
\frac{\partial \Pi}{\partial w_{m n}}=0
$$
by:

This yields a system of algebraic equations: given generally

$$
\sum_{m=1}^{\infty} \sum_{n=1}^{\infty} k_{m n} w_{m n}=\sum_{m=1}^{\infty} \sum_{n=1}^{\infty} F_{m n}
$$

where $k_{m n}$ are stiffness and $F_{m n}$ are force terms and

$$
\begin{aligned}
& k_{m n}=\iint_{R}\left[X_{m}^{\prime \prime} Y_{n} X_{k}^{\prime \prime} Y_{l}+X_{m} X_{k} Y_{n}^{\prime \prime} Y_{l}^{\prime \prime}+X_{m} X_{k}^{\prime \prime} Y_{n} Y_{l}^{\prime \prime}+X_{m}^{\prime \prime} X_{k} Y_{n}^{\prime \prime} Y_{l}\right. \\
& \left.+2(1-\mu)\left(X_{m}^{\prime} Y_{n}^{\prime} X_{k}^{\prime} Y_{l}^{\prime}-X_{m}^{\prime \prime} X_{k} Y_{n}^{\prime \prime} Y_{l}\right)\right] d x d y \\
& F_{m n}=\frac{1}{D} \iint_{R} p(x, y) X_{m} Y_{n} d x d y
\end{aligned}
$$

$k_{m n}=\iint\left[\left(\nabla^{2} X_{m} Y_{n}\right)\left(\nabla^{2} X_{k} Y_{l}\right)-(1-\mu)\left(X_{m}^{\prime \prime} X_{k} Y_{n} Y_{l}^{\prime \prime}\right.\right.$

$\left.\left.+X_{m} X_{k}^{\prime \prime} Y_{n}^{\prime \prime} Y_{l}-2 X_{m}^{\prime} X_{k}^{\prime} Y_{n}^{\prime} Y_{l}^{\prime}\right)\right] d x d y$

An approximation to the displacement field is achieved in the Ritz variational method by a truncation of the Ritz displacement field such that $m$ and $n$ are finite values. Thus, $m$ $=1,2, \ldots M ; n=1,2, \ldots N$.

Consequently, the matrix equation becomes a finite system of algebraic equations in terms of the unknown displacement parameters $w_{m n}$, which can be solved by matrix inversion, and other methods of matrix algebra.

\section{APPLICATION OF THE RITZ VARIATIONAL METHOD}

Consider a rectangular Kirchhoff plate with isotropic, homogeneous material properties and all edges simply supported. The plate is subjected to a hydrostatic load distribution of intensity $q(x)$; where $q_{z}(x)=q_{0} x / a$ where $q_{0}$ is the intensity at $x=a$, and $q(x)$ acts over the entire area of the plate surface. The plate is defined by the Cartesian coordinate system shown in Figure 1, with the origin chosen at a corner of the plate. The plate domain is defined by $0 \leq x \leq a, 0 \leq y \leq b$

where $a$, and $b$ are the length and width of the plate, respectively. The geometric and force boundary conditions are

$$
\begin{aligned}
& w(x=0, y)=w(x=a, y)=0 \\
& w(x, y=0)=w(x, y=b)=0 \\
& w_{x x}(x=0, y)=w_{x x}(x=a, y)=0 \\
& w_{y y}(x, y=0)=w_{y y}(x, y=b)=0
\end{aligned}
$$

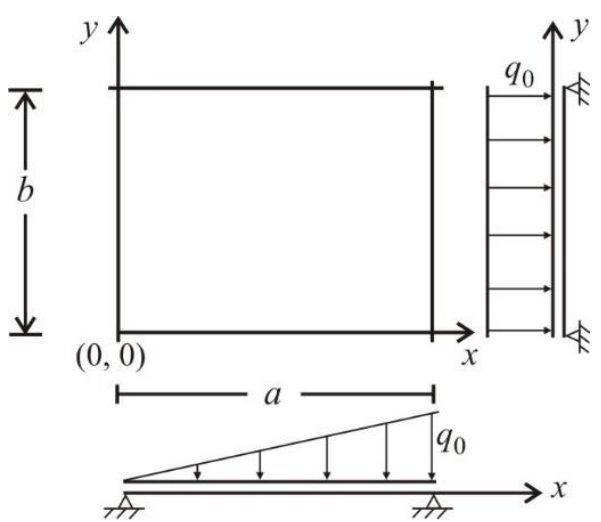

Figure 1. Rectangular Kirchhoff plate with simply supported edges carrying hydrostatic load in the $x$-coordinate direction

A suitable displacement field that satisfies all the geometric and force boundary conditions of simple supports at the four edges is given by:

$w(x, y)=\sum_{m=1}^{\infty} \sum_{n=1}^{\infty} C_{m n} \sin \frac{m \pi x}{a} \sin \frac{n \pi y}{b}$

where $m=1,2,3, \ldots \infty$

$n=1,2,3, \ldots \infty$

$C_{m n}$ are the generalized displacement coordinates of the displacement function which are unknowns, and which we seek to determine in order to find the deflection function $w(x$, $y$ ). The total potential energy functional $\Pi$ for a Kirchhoff plate made with homogeneous isotropic material is given by

$\Pi=U_{b}+V$

where $U_{b}$ is the strain energy functional for bending of the Kirchhoff plate and $V$ is the potential energy functional due to the applied transverse load $q(x, y)$.

$U_{b}=\frac{D}{2} \iint_{R_{x y}}\left[\left(\nabla^{2} w\right)^{2}+2(1-\mu)\left(w_{x y}^{2}-w_{x x} w_{y y}\right)\right] d x d y$

where $\nabla^{2}=\frac{\partial^{2}}{\partial x^{2}}+\frac{\partial}{\partial y^{2}}$

$w_{x y}=\frac{\partial^{2} w}{\partial x \partial y}$

$w_{x x}=\frac{\partial^{2} w}{\partial x^{2}}$

$w_{y y}=\frac{\partial^{2} w}{\partial y^{2}}$

$D$ is the plate flexural rigidity, and $\mu$ is the Poisson's ratio of the plate material.

$R_{x y}$ is the two dimensional domain of the plate defined by $0 \leq x \leq a, 0 \leq y \leq b, z=0$ 
$V=-\iint_{R_{x y}} q(x, y) w(x, y) d x d y$

$U_{b}=\frac{D}{2} \iint\left(w_{x x}+w_{y y}\right)^{2} d x d y+\frac{D}{2} \iint 2(1-\mu)\left(w_{x y}^{2}-w_{x x} w_{y y}\right) d x d y$

$U_{b}=\frac{D}{2} \iint_{R_{x y}}\left(w_{x x}+w_{y y}\right)^{2} d x d y+D(1-\mu) \iint_{R_{x y}}\left(w_{x y}^{2}-w_{x x} w_{y y}\right) d x d y$

$U_{b}=\frac{D}{2} \iint_{R_{x y}}\left(\nabla^{2} w\right)^{2} d x d y+D(1-\mu) \iint_{R_{x y}}\left(w_{x y}^{2}-w_{x x} w_{y y}\right) d x d y$

$U_{b}=\frac{D}{2} I_{1}+D(1-\mu) I_{2}$

where $I_{1}=\iint_{R_{x y}}\left(\nabla^{2} w\right)^{2} d x d y$

$$
I_{2}=\iint_{R_{x y}}\left(w_{x y}^{2}-w_{x x} w_{y y}\right)^{2} d x d y
$$

where $w_{x y}^{2}-w_{x x} w_{y y}$ is called the Gaussian curvature of the plate.

$\left(\nabla^{2} w\right)^{2}=\left(\nabla^{2} \sum_{m}^{\infty} \sum_{n}^{\infty} C_{m n} \sin \frac{m \pi x}{a} \sin \frac{n \pi y}{b}\right)^{2}$

$\left(\nabla^{2} w\right)^{2}=\left(\sum_{m}^{\infty} \sum_{n}^{\infty} \nabla^{2} C_{m n} \sin \frac{m \pi x}{a} \sin \frac{n \pi y}{b}\right)^{2}$

$\left(\nabla^{2} w\right)^{2}=\left(\sum_{m}^{\infty} \sum_{n}^{\infty}-\left(\left(\frac{m \pi}{a}\right)^{2}+\left(\frac{n \pi}{b}\right)^{2}\right) C_{m n} \sin \frac{m \pi x}{a} \sin \frac{n \pi y}{b}\right)^{2}$

$\left(\nabla^{2} w\right)^{2}=\left(-\pi^{2} \sum_{m}^{\infty} \sum_{n}^{\infty}\left(\frac{m^{2}}{a^{2}}+\frac{n^{2}}{b^{2}}\right) C_{m n} \sin \frac{m \pi x}{a} \sin \frac{n \pi y}{b}\right)^{2}$

$\left(\nabla^{2} w\right)^{2}=\pi^{4}\left(\sum_{m}^{\infty} \sum_{n}^{\infty}\left(\frac{m^{2}}{a^{2}}+\frac{n^{2}}{b^{2}}\right) C_{m n} \sin \frac{m \pi x}{a} \sin \frac{n \pi y}{b}\right)^{2}$

$\left(\nabla^{2} w\right)^{2}=\pi^{4}\left\{\left[\sum_{m}^{\infty} \sum_{n}^{\infty}\left(\frac{m^{2}}{a^{2}}+\frac{n^{2}}{b^{2}}\right)^{2} C_{m n}^{2} \sin ^{2} \frac{m \pi x}{a} \sin ^{2} \frac{n \pi y}{b}\right]\right.$

$+\sum_{m}^{\infty} \sum_{n}^{\infty} \sum_{m^{\prime}}^{\infty} \sum_{n^{\prime}}^{\infty}\left(\frac{m^{2}}{a^{2}}+\frac{n^{2}}{b^{2}}\right)\left(\frac{m^{\prime 2}}{a^{2}}+\frac{n^{\prime 2}}{b^{2}}\right) C_{m n} C_{m^{\prime} n^{\prime}} \times$

$\left.\sin \frac{m \pi x}{a} \sin \frac{n \pi y}{b} \sin \frac{m^{\prime} \pi x}{a} \sin \frac{n^{\prime} \pi y}{b}\right\}$
$I_{1}=\int_{0}^{a} \int_{0}^{b} \pi^{4}\left\{\sum_{m}^{\infty} \sum_{n}^{\infty}\left(\frac{m^{2}}{a^{2}}+\frac{n^{2}}{b^{2}}\right)^{2} C_{m n}^{2} \sin ^{2} \frac{m \pi x}{a} \sin ^{2} \frac{n \pi y}{b}\right.$

$+\sum_{m}^{\infty} \sum_{n}^{\infty} \sum_{m^{\prime}}^{\infty} \sum_{n^{\prime}}^{\infty}\left(\frac{m^{2}}{a^{2}}+\frac{n^{2}}{b^{2}}\right)\left(\frac{m^{\prime 2}}{a^{2}}+\frac{n^{\prime 2}}{b^{2}}\right) C_{m n} C_{m^{\prime} n^{\prime}} \times$

$\left.\sin \frac{m \pi x}{a} \sin \frac{n \pi y}{b} \sin \frac{m^{\prime} \pi x}{a} \sin \frac{n^{\prime} \pi y}{b}\right\} d x d y$

The sinusoidal functions $\sin \frac{m \pi x}{a}$ and $\sin \frac{n \pi y}{b}$ are orthogonal functions, and from the properties of orthogonal functions we obtain

$$
\begin{aligned}
& \int_{0}^{a} \sin \frac{m \pi x}{a} \sin \frac{m^{\prime} \pi x}{a} d x=0 \text { for } m \neq m^{\prime} \\
& \int_{0}^{b} \sin \frac{n \pi x}{b} \sin \frac{n^{\prime} \pi y}{b} d y=0 \text { for } n \neq n^{\prime}
\end{aligned}
$$

The orthogonality properties of the sinusoidal basis functions of the plate lead to the vanishing of the second term in the expression for the integral $I_{1}$ and the simplification of $I_{1}$ to become:

$$
\begin{aligned}
& I_{1}=\pi^{4} \int_{0}^{a} \int_{0}^{b} \sum_{m}^{\infty} \sum_{n}^{\infty}\left(\frac{m^{2}}{a^{2}}+\frac{n^{2}}{b^{2}}\right)^{2} C_{m n}^{2} \sin ^{2} \frac{m \pi x}{a} \sin ^{2} \frac{n \pi y}{b} d x d y \\
& I_{1}=\pi^{4} \sum_{m}^{\infty} \sum_{n}^{\infty}\left(\frac{m^{2}}{a^{2}}+\frac{n^{2}}{b^{2}}\right)^{2} C_{m n}^{2} \int_{0}^{a} \int_{0}^{b} \sin ^{2} \frac{m \pi x}{a} \sin ^{2} \frac{n \pi y}{b} d x d y \\
& I_{1}=\pi^{4} \sum_{m}^{\infty} \sum_{n}^{\infty}\left(\frac{m^{2}}{a^{2}}+\frac{n^{2}}{b^{2}}\right)^{2} C_{m n}^{2} \int_{0}^{a} \sin ^{2} \frac{m \pi x}{a} d x \int_{0}^{b} \sin ^{2} \frac{n \pi y}{b} d y \\
& I_{1}=\pi^{4} \sum_{m}^{\infty} \sum_{n}^{\infty}\left(\frac{m^{2}}{a^{2}}+\frac{n^{2}}{b^{2}}\right)^{2} C_{m n}^{2} \frac{a}{2} \frac{b}{2} \\
& I_{1}=\frac{\pi^{4} a b}{4} \sum_{m}^{\infty} \sum_{n}^{\infty}\left(\frac{m^{2}}{a^{2}}+\frac{n^{2}}{b^{2}}\right)^{2} C_{m n}^{2}
\end{aligned}
$$

Similarly,

$$
\begin{aligned}
& I_{2}=\iint\left(w_{x y}^{2}-w_{x x} w_{y y}\right) d x d y \\
& w_{x y}^{2}=\left(\pi^{2} \sum_{m}^{\infty} \sum_{n}^{\infty} \frac{m n}{a b} C_{m n} \cos \frac{m \pi x}{a} \cos \frac{n \pi y}{b}\right)^{2}
\end{aligned}
$$

Hence, 


$$
\begin{aligned}
& w_{x y}^{2}=\pi^{4} \sum_{m}^{\infty} \sum_{n}^{\infty}\left(\frac{m n}{a b}\right)^{2} C_{m n}^{2} \cos ^{2} \frac{m \pi x}{a} \cos ^{2} \frac{n \pi y}{b} \\
& w_{x x} w_{y y}=\left(\sum_{m}^{\infty} \sum_{n}^{\infty}-\left(\frac{m \pi}{a}\right)^{2} C_{m n} \sin \frac{m \pi x}{a} \sin \frac{n \pi y}{b}\right) \times 1 \\
& \left(\sum_{m}^{\infty} \sum_{n}^{\infty}-\left(\frac{n \pi}{b}\right)^{2} C_{m n} \sin \frac{m \pi x}{a} \sin \frac{n \pi y}{b}\right) \\
& =\sum_{m}^{\infty} \sum_{n}^{\infty} \pi^{4} \frac{m^{2} n^{2}}{a^{2} b^{2}} C_{m n}^{2} \sin ^{2} \frac{m \pi x}{a} \sin ^{2} \frac{n \pi y}{b} \\
& w_{x y}^{2}-w_{x x} w_{y y}=\pi^{4} \sum_{m}^{\infty} \sum_{n}^{\infty}\left(\frac{m n}{a b}\right)^{2} C_{m n}^{2}\left(\cos ^{2} \frac{m \pi x}{a} \cos ^{2} \frac{n \pi y}{b}-\sin ^{2} \frac{m \pi x}{a} \sin ^{2} \frac{n \pi y}{b}\right) \\
& I_{2}=\iint_{0}\left(w_{x y}^{2}-w_{x x} w_{y y}\right) d x d y \\
& I_{2}=\iint_{\pi^{4}} \sum_{m}^{\infty} \sum_{n}^{\infty}\left(\frac{m n}{a b}\right)^{2} C_{m n}^{2}\left(\cos ^{2} \frac{m \pi x}{a} \cos ^{2} \frac{n \pi y}{b}-\sin ^{2} \frac{m \pi x}{a} \sin ^{2} \frac{n \pi y}{b}\right) d x d y \\
& U_{b}=\frac{D}{2} I_{1}=\frac{D}{2} \frac{\pi^{4} a b}{4} \sum_{m}^{\infty} \sum_{m}^{\infty} \sum_{n}^{\infty}\left(\frac{m n}{a b}\right)^{2} C_{m n}^{2}\left(\frac{m^{2}}{a^{2}}+\frac{n^{2}}{b^{2}}\right)_{0}^{a b} \int_{0}^{4} C_{m}^{2} \cos ^{2} \frac{m \pi x}{a} \cos ^{2} \frac{n \pi y}{b} d x d y \\
& I_{2}=0 \\
& -\int_{0} \int_{0}^{b} \sin ^{2} \frac{m \pi x}{a} \sin ^{2} \frac{n \pi y}{b} d x d y \\
& I_{2}
\end{aligned}
$$

The potential energy functional due to the applied distributed transverse load $q(x, y)$ is

$$
\begin{aligned}
& V_{e}=-\int_{0}^{a} \int_{0}^{b} q(x, y) w(x, y) d x d y \\
& =-\int_{0}^{a} \int_{0}^{b} \frac{q_{0} x}{a} \sum_{m}^{\infty} \sum_{n}^{\infty} C_{m n} \sin \frac{m \pi x}{a} \sin \frac{n \pi y}{b} d x d y \\
& =-\frac{q_{0}}{a} \sum_{m}^{\infty} \sum_{n}^{\infty} C_{m n} \int_{0}^{a} \int_{0}^{b} x \sin \frac{m \pi x}{a} \sin \frac{n \pi y}{b} d x d y \\
& =-\frac{q_{0}}{a} \sum_{m}^{\infty} \sum_{n}^{\infty} C_{m n} I_{3}
\end{aligned}
$$

where $I_{3}=\int_{0}^{a} \int_{0}^{b} x \sin \frac{m \pi x}{a} \sin \frac{n \pi y}{b} d x d y$

$I_{3}=\int_{0}^{a} x \sin \frac{m \pi x}{a} d x \int_{0}^{b} \sin \frac{n \pi y}{b} d y$

$I_{3}=\left(-\left[\left(\frac{a}{m \pi}\right)^{2} \sin \frac{m \pi x}{a}-\frac{a x}{m \pi} \cos \frac{m \pi x}{a}\right]_{0}^{a}\right)\left[\frac{-b}{n \pi} \cos \frac{n \pi y}{b}\right]_{0}^{b}$

$I_{3}=\left(-\left[\left(\frac{a}{m \pi}\right)^{2} \sin m \pi-\frac{a^{2}}{m \pi} \cos m \pi\right]\right)\left(\frac{-b}{n \pi}(\cos n \pi-1)\right)$

$V_{e}=-\frac{q_{0} C_{m n}}{a} \sum_{m=1}^{\infty} \frac{(-1)^{m+1} a^{2}}{m \pi} \sum_{n=1}^{\infty} \frac{2 b}{n \pi}$

$V_{e}=\frac{-2 q_{0} a b C_{m n}}{\pi^{2}} \sum_{m=1}^{\infty} \frac{(-1)^{m+1}}{m} \sum_{n=1}^{\infty} \frac{1}{n}$

where $m=1,2,3,4,5, \ldots$

$n=1,3,5,7,9, \ldots$

The total potential energy functional is given in Equation (10)

$\Pi=\frac{\pi^{4} a b D}{8} \sum_{m}^{\infty} \sum_{n}^{\infty}\left(\frac{m^{2}}{a^{2}}+\frac{n^{2}}{b^{2}}\right)^{2} C_{m n}^{2}-\frac{2 q_{0} a b C_{m n}}{\pi^{2}} \sum_{m}^{\infty} \sum_{n}^{\infty} \frac{(-1)^{m+1}}{m n}$
$m=1,2,3, \ldots ; n=1,3,5,7, \ldots$

$\Pi=f\left(C_{m n}\right)$

For extremum of $\Pi$ with respect to the generalized coordinates, the principle of minimization of the total potential energy functional with respect to $C_{m n}$ requires that:

$$
\frac{\partial \Pi}{\partial C_{m n}}=0
$$

$\frac{\partial\left(U+V_{e}\right)}{\partial C_{m n}}=\frac{\partial U}{\partial C_{m n}}+\frac{\partial V_{e}}{\partial C_{m n}}=0$

$\frac{\partial \Pi}{\partial C_{m n}}=\frac{\pi^{4} a b D}{8} \sum_{m}^{\infty} \sum_{n}^{\infty}\left(\frac{m^{2}}{a^{2}}+\frac{n^{2}}{b^{2}}\right)^{2} 2 C_{m n}-\frac{2 q_{0} a b}{\pi^{2}} \sum_{m}^{\infty} \sum_{n}^{\infty} \frac{(-1)^{m+1}}{m n}=0$

$\frac{\pi^{4} a b D}{4} \sum_{m}^{\infty} \sum_{n}^{\infty}\left(\frac{m^{2}}{a^{2}}+\frac{n^{2}}{b^{2}}\right)^{2} C_{m n}=\frac{2 q_{0} a b}{\pi^{2}} \sum_{m}^{\infty} \sum_{n}^{\infty} \frac{(-1)^{m+1}}{m n}$

$C_{m n}=\frac{\frac{2 q_{0} a b}{\pi^{2}} \frac{(-1)^{m+1}}{m n}}{\frac{\pi^{4} a b D}{4}\left(\frac{m^{2}}{a^{2}}+\frac{n^{2}}{b^{2}}\right)^{2}}$ 


$$
C_{m n}=\frac{8 q_{0}(-1)^{m+1}}{\pi^{6} D m n\left(\frac{m^{2}}{a^{2}}+\frac{n^{2}}{b^{2}}\right)^{2}}
$$

Hence,

$$
w(x, y)=\sum_{m}^{\infty} \sum_{n}^{\infty} \frac{8 q_{0}(-1)^{m+1} \sin \frac{m \pi x}{a} \sin \frac{n \pi y}{b}}{\pi^{6} D m n\left(\frac{m^{2}}{a^{2}}+\frac{n^{2}}{b^{2}}\right)^{2}}(63)
$$$$
w(x, y)=\frac{8 q_{0}}{\pi^{6} D} \sum_{m}^{\infty} \sum_{n}^{\infty} \frac{\sin \frac{m \pi x}{a} \sin \frac{n \pi y}{b}(-1)^{m+1}}{m n\left(\frac{m^{2}}{a^{2}}+\frac{n^{2}}{b^{2}}\right)^{2}}
$$

Let $b / a=\alpha$

$$
w(x, y)=\frac{8 q_{0} a^{4} \alpha^{4}}{\pi^{6} D} \sum_{m}^{\infty} \sum_{n}^{\infty} \frac{(-1)^{m+1} \sin \frac{m \pi x}{a} \sin \frac{n \pi y}{b}}{m n\left(m^{2} \alpha^{2}+n^{2}\right)^{2}}
$$

$w(x, y)=\left(\frac{8}{\pi^{6} D} \sum_{m}^{\infty} \sum_{n}^{\infty} \frac{(-1)^{m+1} \sin \frac{m \pi x}{a} \sin \frac{n \pi y}{b}}{m n\left(m^{2} \alpha^{2}+n^{2}\right)^{2}}\right) q_{0} b^{4}$

Let $a / b=\alpha_{1}$

$w(x, y)=\left(\frac{8}{\pi^{6} D} \sum_{m}^{\infty} \sum_{n}^{\infty} \frac{(-1)^{m+1} \sin \frac{m \pi x}{a} \sin \frac{n \pi y}{b}}{m n\left(m^{2}+n^{2} \alpha_{1}^{2}\right)^{2}}\right) q_{0} a^{4}$

For square plates, $\alpha=1$,

$w(x, y)=\frac{8 q_{0} a^{4}}{\pi^{6} D} \sum_{m}^{\infty} \sum_{n}^{\infty} \frac{(-1)^{m+1} \sin \frac{m \pi x}{a} \sin \frac{n \pi y}{b}}{m n\left(m^{2}+n^{2}\right)^{2}}$

Deflection at the plate center $(x=a / 2, y=b / 2)$

The deflection at the plate center is given by

$w\left(x=\frac{a}{2}, y=\frac{b}{2}\right)=\left(\frac{8}{\pi^{6} D} \sum_{m}^{\infty} \sum_{n}^{\infty} \frac{(-1)^{m+1} \sin \frac{m \pi}{2} \sin \frac{n \pi}{2}}{m n\left(m^{2}+n^{2} \alpha_{1}^{2}\right)^{2}}\right) q_{0} a^{4}$

$=\left(\frac{8}{\pi^{6} D} \sum_{m}^{\infty} \sum_{n}^{\infty} \frac{(-1)^{m+1} \sin \frac{m \pi}{2} \sin \frac{n \pi}{2}}{m n\left(m^{2} \alpha^{2}+n^{2}\right)^{2}}\right) q_{0} b^{4}$

For square plates, the center deflection is given by

$$
w_{c}=w(x=a / 2, y=b / 2)=\frac{8 q_{0} a^{4}}{\pi^{6} D} \sum_{m}^{\infty} \sum_{n}^{\infty} \frac{(-1)^{m+1} \sin \frac{m \pi}{2} \sin \frac{n \pi}{2}}{m n\left(m^{2}+n^{2}\right)^{2}}
$$

$$
w_{c}=\frac{8 q_{0} a^{4}}{\pi^{6} D} \sum_{m}^{\infty} \sum_{n}^{\infty} \frac{(-1)^{m+1}(-1)^{\frac{m+n-2}{2}}}{m n\left(m^{2}+n^{2}\right)^{2}}
$$

For $m=1, n=1$, and square plate, $\alpha=1$

$$
w_{c}=\frac{2}{\pi^{6}} \frac{q_{0} a^{4}}{D}=0.002038 \frac{q_{0} a^{4}}{D}
$$

For square plates, summing up to $m=n=3$, the center deflection is obtained as $w_{c}=0.002024 \frac{q_{0} a^{4}}{D}$ which is not significantly different from the center deflection for a one term Ritz variational solution.

\section{Bending moment distribution}

The bending moment distribution $M_{x x}$ and $M_{y y}$ are given by:

$$
\begin{aligned}
& M_{x x}=-D\left(\frac{\partial^{2} w}{\partial x^{2}}+\mu \frac{\partial^{2} w}{\partial y^{2}}\right) \\
& M_{y y}=-D\left(\frac{\partial^{2} w}{\partial y^{2}}+\mu \frac{\partial^{2} w}{\partial x^{2}}\right)
\end{aligned}
$$

From Equation (66),

$$
w_{x x}=\frac{\partial^{2} w}{\partial x^{2}}=\frac{8 q b^{4}}{\pi^{6} D} \sum_{m}^{\infty} \sum_{n}^{\infty} \frac{-\left(\frac{m \pi}{a}\right)^{2} \sin \frac{m \pi x}{a} \sin \frac{n \pi y}{b}(-1)^{m+1}}{m n\left(m^{2} \alpha^{2}+n^{2}\right)^{2}}
$$

$$
w_{y y}=\frac{\partial^{2} w}{\partial y^{2}}=\frac{8 q b^{4}}{\pi^{6} D} \sum_{m}^{\infty} \sum_{n}^{\infty} \frac{\left(\frac{n \pi}{b}\right)^{2} \sin \frac{m \pi x}{a} \sin \frac{n \pi y}{b}(-1)^{m+1}}{m n\left(m^{2} \alpha^{2}+n^{2}\right)^{2}}
$$

Thus,

$$
\begin{aligned}
& M_{x x}=\frac{8 q b^{4}}{\pi^{6}} \sum_{m}^{\infty} \sum_{n}^{\infty} \frac{(-1)^{m+1}\left(\left(\frac{m \pi}{a}\right)^{2}+\mu\left(\frac{n \pi}{b}\right)^{2}\right) \sin \frac{m \pi x}{a} \sin \frac{n \pi y}{b}}{m n\left(m^{2} \alpha^{2}+n^{2}\right)^{2}} \\
& \left(\frac{m \pi}{a}\right)^{2}+\mu\left(\frac{n \pi}{b}\right)^{2}=\pi^{2}\left(\frac{m^{2}}{a^{2}}+\mu \frac{n^{2}}{b^{2}}\right) \\
& =\pi^{2}\left(\frac{m^{2}}{b^{2}}+\mu \frac{n^{2}}{b^{2}}\right)
\end{aligned}
$$




$$
\begin{aligned}
& =\pi^{2}\left(\frac{m^{2} \alpha^{2}}{b^{2}}+\mu \frac{n^{2}}{b^{2}}\right) \\
& =\pi^{2}\left(\frac{m^{2} \alpha^{2}+\mu n^{2}}{b^{2}}\right)
\end{aligned}
$$

$M_{x x}=\frac{8 q b^{4} \pi^{2}}{\pi^{6} b^{2}} \sum_{m}^{\infty} \sum_{n}^{\infty} \frac{(-1)^{m+1}\left(m^{2} \alpha^{2}+\mu n^{2}\right) \sin \frac{m \pi x}{a} \sin \frac{n \pi y}{b}}{m n\left(m^{2} \alpha^{2}+n^{2}\right)^{2}}$

$=\frac{8 q b^{2}}{\pi^{4}} \sum_{m}^{\infty} \sum_{n}^{\infty} \frac{(-1)^{m+1}\left(m^{2} \alpha^{2}+\mu n^{2}\right) \sin \frac{m \pi x}{a} \sin \frac{n \pi y}{b}}{m n\left(m^{2} \alpha^{2}+n^{2}\right)^{2}}$

At the center, $x=a / 2, y=b / 2$

$M_{x x}(x=a / 2, y=b / 2)=\frac{8 q_{0} b^{2}}{\pi^{4}} \sum_{m}^{\infty} \sum_{n}^{\infty} \frac{(-1)^{m+1}\left(m^{2} \alpha^{2}+\mu n^{2}\right) \sin \frac{m \pi}{2} \sin \frac{n \pi}{2}}{m n\left(m^{2} \alpha^{2}+n^{2}\right)^{2}}$

$=\left(\frac{8}{\pi^{4}} \sum_{m}^{\infty} \sum_{n}^{\infty} \frac{(-1)^{m+1}\left(m^{2} \alpha^{2}+\mu n^{2}\right)(-1)^{\frac{m+n}{2}-1}}{m n\left(m^{2} \alpha^{2}+n^{2}\right)^{2}}\right) q_{0} b^{2}$

$M_{x x}=\beta_{x x} q_{0} b^{2}$

$\beta_{x x}$ is the bending moment coefficient for $M_{x x}$ and is given by:

$\beta_{x x}=\frac{8}{\pi^{4}} \sum_{m}^{\infty} \sum_{n}^{\infty} \frac{(-1)^{m+1}(-1)^{\frac{m+n}{2}-1}\left(m^{2} \alpha^{2}+\mu n^{2}\right)}{m n\left(m^{2} \alpha^{2}+n^{2}\right)^{2}}$

Similarly,

$M_{y y}=\frac{8 q_{0} b^{4}}{\pi^{6} D} \sum_{m}^{\infty} \sum_{n}^{\infty} \frac{(-1)^{m+1}\left(\left(\frac{n \pi}{b}\right)^{2}+\mu\left(\frac{m \pi}{a}\right)^{2}\right) \sin \frac{m \pi x}{a} \sin \frac{n \pi y}{b}}{m n\left(m^{2} \alpha^{2}+n^{2}\right)^{2}}$

$$
\begin{aligned}
& \left(\frac{n \pi}{b}\right)^{2}+\mu\left(\frac{m \pi}{a}\right)^{2}=\frac{\pi^{2} n^{2}}{b^{2}}+\mu \frac{\pi^{2} m^{2}}{a^{2}}=\pi^{2}\left(\frac{n^{2}}{b^{2}}+\mu \frac{m^{2}}{a^{2}}\right) \\
& =\pi^{2}\left(\frac{n^{2}}{b^{2}}+\mu \frac{m^{2} \alpha^{2}}{b^{2}}\right)=\frac{\pi^{2}}{b^{2}}\left(n^{2}+\mu m^{2} \alpha^{2}\right)
\end{aligned}
$$

$M_{y y}=\frac{8 q_{0} b^{4} \pi^{2}}{\pi^{6} b^{2}} \sum_{m}^{\infty} \sum_{n}^{\infty} \frac{(-1)^{m+1}\left(n^{2}+\mu m^{2} \alpha^{2}\right) \sin \frac{m \pi x}{a} \sin \frac{n \pi y}{b}}{m n\left(m^{2} \alpha^{2}+n^{2}\right)^{2}}$

$=\frac{8 q_{0} b^{2}}{\pi^{4}} \sum_{m}^{\infty} \sum_{n}^{\infty} \frac{(-1)^{m+1}\left(n^{2}+\mu m^{2} \alpha^{2}\right) \sin \frac{m \pi x}{a} \sin \frac{n \pi y}{b}}{m n\left(m^{2} \alpha^{2}+n^{2}\right)^{2}}$

At the center, $x=a / 2, y=b / 2$

$$
\begin{aligned}
& M_{x x}(x=a / 2, y=b / 2)=\frac{8 q_{0} b^{2}}{\pi^{4}} \sum_{m}^{\infty} \sum_{n}^{\infty} \frac{(-1)^{m+1}\left(n^{2}+\mu m^{2} \alpha^{2}\right) \sin \frac{m \pi}{2} \sin \frac{n \pi}{2}}{m n\left(m^{2} \alpha^{2}+n^{2}\right)^{2}} \\
& =\left(\frac{8}{\pi^{4}} \sum_{m}^{\infty} \sum_{n}^{\infty} \frac{(-1)^{m+1}(-1)^{\frac{m+n}{2}-1}\left(n^{2}+\mu m^{2} \alpha^{2}\right)}{m n\left(m^{2} \alpha^{2}+n^{2}\right)^{2}}\right) q_{0} b^{2} \\
& M_{y y}=\beta_{x x} q_{0} b^{2}
\end{aligned}
$$

$\beta_{y y}$ is the bending moment coefficient for $M_{y y}$ and is given by:

$$
\beta_{y y}=\frac{8}{\pi^{4}} \sum_{m}^{\infty} \sum_{n}^{\infty} \frac{(-1)^{m+1}(-1)^{\frac{m+n}{2}-1}\left(n^{2}+\mu m^{2} \alpha^{2}\right)}{m n\left(m^{2} \alpha^{2}+n^{2}\right)^{2}}
$$

The deflection coefficients for the center of the plate for $m$ $=1, n=1$ in the Ritz variational solution are tabulated in Table 1 , for various aspect ratios of the rectangular plate.

The deflection coefficient for the center deflection of the plate $m=1,3 ; n=1,3$ in the Ritz variational solution are tabulated in Table 2, for various plate aspect ratios.

Table 1. Deflection coefficients at center of simply supported Kirchhoff plate under hydrostatic load (for $m=1, n=1$ ) in the Ritz variational method

\begin{tabular}{|l|l|}
\hline$\alpha=b / a$ & $w_{c}=F_{1}(\alpha)^{q_{0}} b^{4} / D$ \\
\hline 1 & $2.0803 \times 10^{-3}$ \\
\hline 1.1 & $2.4945 \times 10^{-3}$ \\
\hline 1.2 & $2.8983 \times 10^{-3}$ \\
\hline 1.3 & $3.2844 \times 10^{-3}$ \\
\hline 1.4 & $3.6485 \times 10^{-3}$ \\
\hline 1.5 & $3.9883 \times 10^{-3}$ \\
\hline 1.6 & $4.3030 \times 10^{-3}$ \\
\hline 1.7 & $4.5929 \times 10^{-3}$ \\
\hline
\end{tabular}




\begin{tabular}{|l|l|}
\hline 1.8 & $4.8590 \times 10^{-3}$ \\
\hline 1.9 & $5.1027 \times 10^{-3}$ \\
\hline 2 & $5.3256 \times 10^{-3}$ \\
\hline 3 & $6.7402 \times 10^{-3}$ \\
\hline 4 & $7.3711 \times 10^{-3}$ \\
\hline 5 & $7.6935 \times 10^{-3}$ \\
\hline$\infty$ & $8.3213 \times 10^{-3}$ \\
\hline
\end{tabular}

Table 2. Deflection coefficients at center of plate simply supported under hydrostatic load (for $m=1,3 ; n=1,3$ in the Ritz variational method)

\begin{tabular}{|c|c|c|c|}
\hline$\alpha=b / a$ & $F_{1}(\alpha)$ & $\begin{array}{c}\text { Timoshenko and } \\
\text { Woinowsky- } \\
\text { Krieger }\end{array}$ & Relative error (\%) \\
\hline 1.0 & $2.0277 \times 10^{-3}=0.00203$ & 0.00203 & 0 \\
\hline 1.1 & $2.42963 \times 10^{-3}=0.00243$ & 0.00243 & 0 \\
\hline 1.2 & $2.82002 \times 10^{-3}=0.00282$ & 0.00282 & 0 \\
\hline 1.3 & $3.18955 \times 10^{-3}=0.00319$ & 0.00319 & 0 \\
\hline 1.4 & $3.53307 \times 10^{-3}=0.00353$ & 0.00353 & 0 \\
\hline 1.5 & $3.8516 \times 10^{-3}=0.00385$ & 0.00386 & -0.26 \\
\hline 1.6 & $4.1412 \times 10^{-3}=0.00414$ & 0.00415 & -0.24 \\
\hline 1.7 & $4.40325 \times 10^{-3}=0.00440$ & 0.00441 & -0.23 \\
\hline 1.8 & $4.6389 \times 10^{-3}=0.00464$ & 0.00465 & -0.22 \\
\hline 1.9 & $4.84972 \times 10^{-3}=0.00485$ & 0.00487 & -0.41 \\
\hline 2 & $5.03791 \times 10^{-3}=0.00504$ & 0.00506 & -0.40 \\
\hline 3 & $6.02264 \times 10^{-3}=0.00602$ & 0.00612 & -1.63 \\
\hline 4 & $6.21131 \times 10^{-3}=0.00621$ & 0.00641 & -3.12 \\
\hline 5 & & 0.00648 & \\
\hline$\infty$ & & 0.00651 & \\
\hline
\end{tabular}

For $m=1, n=1$ in the Ritz variational solution for $\beta_{x x}$, for $\alpha=1, \beta_{x x}=0.02669 \simeq 0.0267, \quad$ and $\quad$ for $\alpha=1.5=b / a, \beta_{x x}=0.0446$

For $m=1,3 ; n=1,3$, in the Ritz variational solution for $\beta_{x x}$, for $\alpha=1, \beta_{x x}=0.02346 \simeq 0.0235$, and for $\alpha=1.5, \beta_{x x}=0.0399499 \simeq 0.03995 \quad$ where $M_{x x}=\beta_{x x} q_{0} a^{2}$

Similarly, $\quad$ for $\quad \alpha=1, m=1,3 ; n=1,3$, $\beta_{y y}=0.02346$ and for $\alpha=1.5, m=1,3 ; n=1,3$,

Table 3. Bending moment coefficients for $m=1,3 ; n=1,3$ in the Ritz variational method

\begin{tabular}{|l|l|l|l|}
\hline$\alpha$ & $\beta_{y y}$ & Exact $\beta_{y y}$ & $\begin{array}{l}\text { Relative error } \\
(\%)\end{array}$ \\
\hline 1.0 & 0.0235 & 0.0239 & -1.67 \\
\hline 1.5 & 0.0240 & 0.0249 & -3.61 \\
\hline
\end{tabular}

$\beta_{y y}=0.02399 \simeq 0.0240$ where $\quad M_{y y}=\beta_{y y} q_{0} a^{2}$.

The results are tabulated in Table 3.

Table 4. Deflection coefficients for $\gamma_{1}$ hydrostatic load on simply supported Kirchhoff plate

\begin{tabular}{|c|c|c|c|}
\hline \multirow{2}{*}{$b / a$} & \multicolumn{3}{|c|}{$w=\gamma_{1} q_{0} a^{4} / \mathrm{Et}^{3}$} \\
\cline { 2 - 4 } & $x=0.25 a$ & $x=0.5 a$ & $x=0.75 a$ \\
\hline 1 & 0.0143 & 0.0221 & 0.0177 \\
\hline 1.2 & 0.0203 & 0.0308 & 0.0241 \\
\hline 1.4 & 0.0257 & 0.0385 & 0.0298 \\
\hline 1.6 & 0.0303 & 0.0453 & 0.0346 \\
\hline 1.8 & 0.0342 & 0.0508 & 0.0385 \\
\hline 2 & 0.0373 & 0.0553 & 0.0417 \\
\hline 3 & 0.0454 & 0.0668 & 0.0498 \\
\hline 4 & 0.0477 & 0.0700 & 0.0521 \\
\hline$\infty$ & 0.0484 & 0.0711 & 0.0529 \\
\hline
\end{tabular}


Convergence to exact solution for the bending moment coefficients $\beta_{x x}$ and $\beta_{y y}$ is achieved using $m=1,3,5,7,9$; $n=1,3,5,7,9$ in the solution obtained by the Ritz variational method. The converged results for deflection and bending moment coefficients for various values of the ratio $b / a$ are presented for $x=0.25 a, x=0.5 a$ and $x=0.75 a$, for the deflection coefficients and for $x=0.5 a$ for the bending moment coefficients and tabulated in Tables 4 and 5 .

Table 5. Bending moment coefficients for hydrostatic load $\gamma=q_{0} x / a$ on simply supported Kirchhoff plate

\begin{tabular}{|c|c|c|}
\hline \multicolumn{1}{|l|}{$b / a$} & $\begin{array}{c}M_{x x}=\beta_{x x} q_{0} a^{2} \\
\beta_{x x}(x=0.5 a)\end{array}$ & $\begin{array}{c}M_{y y}=\beta_{y y} q_{0} a^{2} \\
\beta_{y y}(x=0.5 a)\end{array}$ \\
\hline 1.0 & 0.0239 & 0.0239 \\
\hline 1.2 & 0.0313 & 0.0250 \\
\hline 1.4 & 0.0376 & 0.0253 \\
\hline 1.6 & 0.0431 & 0.0246 \\
\hline 1.8 & 0.0474 & 0.0239 \\
\hline 2 & 0.0508 & 0.0232 \\
\hline 3 & 0.0594 & 0.0202 \\
\hline 4 & 0.0617 & 0.0192 \\
\hline$\infty$ & 0.0625 & 0.0187 \\
\hline
\end{tabular}

\section{DISCUSSIONS}

The Ritz variational method has been successfully applied to the flexural problems of simply supported rectangular Kirchhoff plates subjected to hydrostatic load distribution of intensity $q(x)=q_{0} x / a$ over the entire area $(0 \leq x \leq a, 0 \leq y \leq b)$ of the plate surface. A displacement field was chosen as products of linear combination of displacement shape or coordinate functions that apriori satisfied boundary conditions at the plate contours and unknown generalized displacement parameters, $C_{m n}$, as Equation (9). The total potential energy functional was then determined using the chosen displacement field as Equation (55). It was observed that the total potential energy functional obtained is a double series, and is a function of the generalized displacement coordinates. The principle of minimum total potential energy functional was then applied to obtain the unknown generalized displacement coordinates as Equation (64). Thus, the displacement field was obtained as Equation (64). The plate deflection was expressed in terms of the plate aspect ratios $\alpha$ and $\alpha_{1}$ as Equations (66) and (67) respectively. The plate deflection was also obtained for square Kirchhoff plates as Equation (68). The deflection was found at the center of the plate as Equations (69) and (70), for rectangular Kirchhoff plates, and as Equation (72) for square Kirchhoff plates. Bending moment-displacement relationships Equations (74) and (75) were used to obtain the bending moment distribution on the plate as Equations (84) and (89). The bending moments at the plate center were found as Equations (86) and (95). Equations for the deflections and bending moment distributions were found as double series with rapidly convergent properties. The equations for deflections were more rapidly convergent that the equations for bending moments. The Ritz variational solutions obtained using two terms each of the double series for deflection yielded a relative error of less than $1 \%$ for plates with aspect ratios less than 2. Convergence to the exact solution obtained by Timoshenko and Woinowsky-Krieger, who used Levy's single Fourier series method, was obtained by using three terms of the double series for displacement. Similarly, convergence to the exact solution obtained by Timoshenko and Woinowsky-Krieger was obtained by using five terms of the double series for bending moments. However, reasonably accurate solutions were obtained for both deflection and bending moments by using a few terms each, of the double series obtained using the Ritz variational method.

\section{CONCLUSIONS}

The following conclusions can be made:

(i) the Ritz variational method can be successfully applied to the solution of rectangular Kirchhoff plate bending problems for simply supported ends, and transverse hydrostatic loading distribution over the entire plate area.

(ii) the solutions obtained for the deflection and bending moment distributions are double sine series with rapidly convergent properties, thus making the solutions easily amenable to mathematical analysis

(iii) the solution obtained for the center deflections is a double series, with rapidly convergent properties; and convergence to the exact solution is obtained using three terms each of the double series.

(iv) the solutions obtained for the bending moments $M_{x x}$ and $M_{y y}$ at the plate center are double series with good convergence properties and convergence to the exact solution is obtained using five terms each of the double series

(v) the Ritz variational method yielded exactly identical solutions as the exact solutions obtained by Timoshenko and Woinowsky-Krieger who used the Navier double Fourier sine series method. Hence, the Ritz variational method presented in this work yielded the exact solution for the plate flexure problem analyzed. This is attributable to the fact that the exact deflection shape function was used in the Ritz method presented in this study.

\section{REFERENCES}

[1] Chandrashekhara K. (2011). Theory of Plates. University Press, Hyderabad, India.

[2] Timoshenko S, Woinowsky-Krieger S. (1959). Theory of Plates and Shells, 2nd Edition. McGraw Hill Book Co. New York.

[3] Szilard R. (2014). Theories and Applications of Plates Analysis: Classical, Numerical and Engineering Methods. John Wiley and Sons Inc.

[4] Navier CLMN. (1823). Bulletin des science de la societe philomarhique de Paris.

[5] Poisson SD. (1829). Sur les Equations generales de l'Equilibre et du Mouvement des corps solides elastiques et des fluids. Journal de l'Ecole Polytechnique, 13(20): 1-174.

[6] Poisson SD. (1829). Memoire sur l'Equilibre et le mouvement de corps elastiques. Mem. de l'Acad. Sci 8: 357. 
[7] Kirchhoff G. (1850). Uber die Schwingnngen eigner kreisformigen elastischen sheibe annalen der physic. 157(10): 258-264.

[8] Hencky H. (1913). Der Spannungszustand in Rechteckigen Plattern R. Oldernbourg, Munich, Berlin.

[9] Hencky H. (1947). Uber die Berucksichti gnng der schubuerzerrungen in ebenen plattern Ing Arch 16.

[10] Reissner E. (1945). The effect of transverse shear deformation on the bending of elastic plates. Journal of Applied Mechanics 12: 69-77.

[11] Reissner E. (1944). On the theory of bending of elastic plates. Journal of Mathematics and Physics 23: 184-191.

[12] Mindlin RD. (1951). Influence of rotary inertia and shear on flexural motions of isotropic elastic plates. J. Appl. Mech. 18(1): 31-38.

[13] Shimpi RP. (2002). Refined Plate Theory and its variants. AIAA Journal 40: 137-146.

[14] Levinson M. (1980). An accurate simple theory of statics and dynamics of elastic plates. Mechanics Research Communications 7: 343-350.

[15] Reddy JN. (1984). A refined non linear theory of plates with transverse shear deformation. International Journal of Solids and Structures 20: 881-896.

[16] Leung AYT. (1991). An unconstrained third order theory. Computers and Structures 40(4): 871-874.

[17] Osadebe NN. (1997). Differential equations for small deflection analysis of thin elastic plates possessing extensible middle surface. University of Nigeria Virtual Library.

[18] Suetake Y. (2006). Plate bending analysis by using a modified plate theory. CMES 2(3): 103-110.

[19] Sebastian VK. (1983). An elastic solution for simply supported rectangular plates. Nigerian Journal of Technology (NIJOTECH) 7(1): 11-16.

[20] Ladeveze P. (2002). The exact theory of plate bending. Journal of Elasticity 68(1): 37-71.

[21] Levy M. (1899). Memoire sur la theorie des plaque elastiques planes. Journal de Mathematiques Pures et Appliquees, 3: 219.
[22] Nadai A. (1925). Die Elastichen Platten. SpringerVerlag, Berlin.

[23] Mama BO, Nwoji CU, Ike CC, Onah HN. (2017). Analysis of simply supported rectangular kirchhoff plates by the finite fourier sine transform method. International Journal of Advanced Engineering Research and Science (IJAERS) 4(3): 285-291.

[24] Nwoji CU, Mama BO, Onah HN, Ike CC. (2017). Kantorovich-vlasov method for simply supported rectangular plates under uniformly distributed transverse loads. International Journal of Civil, Mechanical and Energy Science (IJCMES) 3(2): 69-77.

[25] Osadebe NN, Ike CC, Onah HN, Nwoji CU, Okafor FO. (2016). Application of Galerkin-Vlasov Method to the flexural analysis of simply supported rectangular Kirchhoff plates under uniform loads. Nigerian Journal of Technology (NIJOTECH) 35(4): 732-738.

[26] Nwoji CU, Mama BO, Ike CC, Onah HN. (2017). Galerkin-Vlasov method for the flexural analysis of rectangular Kirchhoff plates with clamped and simply supported edges. IOSR Journal of Mechanical and Civil Engineering (IOSRJMCE) 14(2): 61-74.

[27] Ike CC. (2017). Kantorovich-Euler-LagrangeGalerkin's method for bending analysis of thin plates. Nigerian Journal of Technology 36(2): 351-360.

[28] Mama BO, Onah HN, Ike CC, Osadebe NN. (2017). Solution of free harmonic vibration equation of simply supported Kirchhoff plate by Galerkin-Vlasov method. Nigerian Journal of Technology 36(2): 361-365.

[29] Ezeh JC, Ibearugbulem OM, Onyechere CI. (2013). Pure bending analysis of thin rectangular flat plates using ordinary finite difference method. International Journal of Emerging Technology and Advanced Engineering (IJETAE) 3(3).

[30] Aginam CH, Chidolue CA, Ezeagu CA. (2012). Application of direct variational method in the analysis of isotropic thin rectangular plates ARPN. Journal of Engineering and Applied Sciences 7(9). 